\title{
Malformación de Chiari tipo I en un paciente con síndrome de Poland
}

\author{
G. Alcalá-Cerra; R. Sabogal-Barrios; L.M. Niño-Hernández**; J.J. Gutiérrez-Paternina*; L.R. Moscote-Salazar***
} y E. Preciado-Mesa

Sección de Neurocirugía. Facultad de Medicina*. Sección de Patología**. Universidad de Cartagena. Hospital Universitario del Caribe. Cartagena de Indias, Colombia. Servicio de Neurocirugía***. Hospital San Rafael. San Juan del Cesar (Guajira), Colombia.

\section{Resumen}

La malformación de Chiari tipo I y el síndrome de Poland son dos patologías infrecuentes, más aún su presentación simultánea no había sido previamente descrita en la literatura.

Se presenta el caso de un paciente varón de 27 años con antecedentes de síndrome de Poland, quien refirió cefalea y alteraciones motoras de los músculos intrínsecos de la mano izquierda. En los estudios de resonancia magnética de columna cervical fue encontrada la malformación de Chiari tipo I con siringomielia desde C1 a $T 2$, la cual es tratada mediante descompresión del foramen magno, plastia de duramadre y remoción del arco posterior del atlas.

Se presenta una discusión de los mecanismos embriológicos que podrían estar implicados en la coexistencia de estas dos entidades, haciendo énfasis en el rol del mesodermo para-axial.

PALABRAS CLAVE. Malformación de Chiari. Unión cráneo-vertebral. Síndrome de Poland. Siringomielia. Anomalía.

Chiari type I Malformation in a patient with Poland's syndrome

Summary

Chiari malformation type I and Poland's syndrome are two rare diseases and their simultaneous presentation had not been previously described in the literature.

We report the case of a 27 year old male with history of Poland's syndrome, who referred headache and motor impairment of the intrinsic muscles of the left hand. In a cervical spine MR a Chiari I malformation with syringomyelia from $\mathrm{C} 1$ to $\mathrm{T} 2$ was found, which was treated by foramen magnum decompression, dural

Recibido: 9-07-11. Aceptado: 26-07-11. plasty and removal of the posterior arch of the atlas.

A discussion of the embryological mechanisms that might be involved in the coexistence of these two entities is presented, emphasizing the role of para-axial mesoderm.

KEY WORDS. Chiari malformation. Craniovertebral junction. Poland's síndrome. Syringomyelia. Anomaly.

\section{Introducción}

Actualmente son reconocidos cinco tipos de la malformación de Chiari甲. La malformación de Chiari tipo I (MCHI) es sin duda la más frecuente, con una prevalencia de $0.078 \%$ en estudios de resonancia magnética de individuos asintomáticos ${ }^{16}$. Esta se caracteriza por una ubicación anormal de las amígdalas cerebelosas mayor o igual a 5 milímetros bajo el plano del foramen magnum. En su patogénesis han sido propuestas diversas teorías; sin embargo, el desarrollo alterado del mesodermo para-axial (MPA) (que origina los huesos occipitales) es una de la más aceptadas ${ }^{9,17,23}$.

Por su parte, el síndrome de Poland (SP) es una anomalía congénita rara con una prevalencia estimada de 1 por 20.000 a 30.000 habitantes, cuyas manifestaciones varían desde una hipoplasia o agenesia aislada y asintomática del músculo pectoralis major hasta grandes defectos torácicos y pulmonares incompatibles con la vida ${ }^{2,10,11}$. La característica distintiva de esta patología es la deficiencia del músculo pectoralis major ${ }^{2}$.

La presentación simultánea de dos anomalías en los tejidos derivados del MPA ha sido explicada por un fenómeno denominado por Marín-Padilla como insuficiencia mesodérmica primaria; el cual podría generar alteraciones tanto del esqueleto axial, como del tejido muscular voluntario, ya que ambos provienen de la misma capa germinal $^{11,15,17}$.

A continuación se presenta el caso de un paciente con

Abreviaturas. MCHI: malformación de Chiari tipo I. MPA: mesodermo para-axial. SP: síndrome de Poland. 


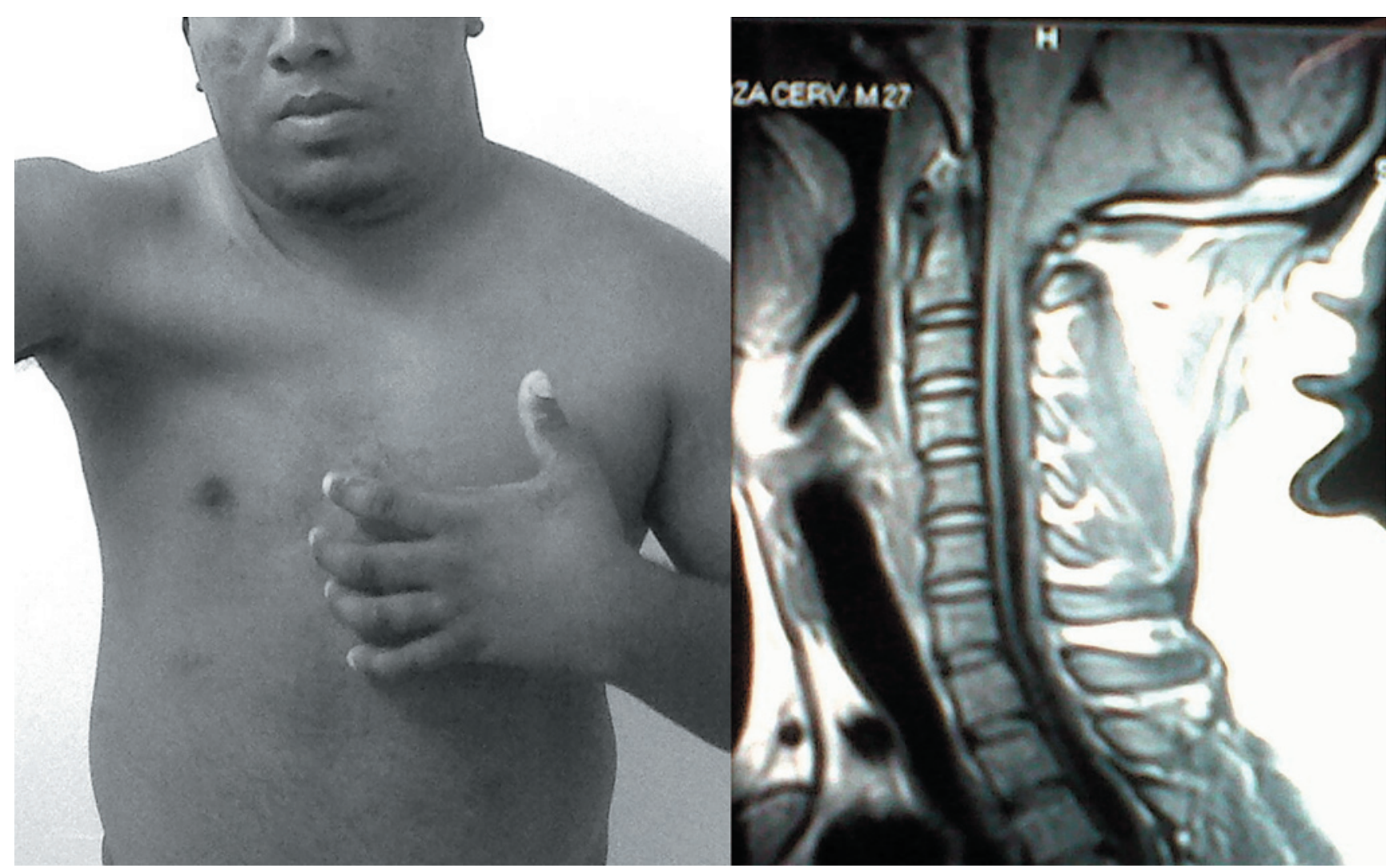

Figura 1. Derecha: imagen de resonancia magnética nuclear de columna cervical, donde es posible visualizar el descenso de las amigdalas del cerebelo y la siringomielia. Izquierda: fotografía que demuestra la ausencia de la porción esternoclavicular del pectoralis major derecho y los cambios tróficos de la musculatura intrínseca de la mano izquierda.

síndrome de Poland y malformación de MCHI sintomática. A nuestro conocimiento, se trata del primer informe de esta asociación.

\section{Presentación del caso}

Un varón de 27 años de edad con antecedentes de síndrome de Poland diagnosticado desde los 4 años de edad acude refiriendo cefalea de predominio occipital asociado a debilidad progresiva y cambios tróficos en la mano izquierda de 8 meses de evolución. Negó disfagia, tos, alteraciones en el patrón del sueño, cambios de la voz, dolor irradiado hacia los miembros superiores u otro síntoma.

$\mathrm{Al}$ examen, se encontró lúcido, orientado y sin alteraciones de las funciones mentales superiores. No se encontraron alteraciones al examen de los pares craneales. La fundoscopia fue normal. Se evidenció hipotrofia y grave debilidad de los músculos interóseos izquierdos y de la eminencia tenar ipsilateral. La fuerza muscular en los demás grupos musculares era normal (Figura 1). Adicionalmente, la sensibilidad térmica y dolorosa en los dermatomas C7, C8 y T1 estaba disminuida. La propio- cepción, así como la sensibilidad vibratoria y al tacto ligero no estaban alteradas. El reflejo tricipital derecho estaba disminuido. No se encontraron signos de neurona motora superior en los miembros superiores ni en los inferiores. La porción clavicular del pectoralis major derecho estaba ausente, sin embargo la fuerza muscular para la aducción y aproximación del brazo era similar a la contralateral.

Una electromiografía con velocidad de conducción nerviosa fue compatible con una lesión de la raíz C8. La resonancia magnética de columna cervical demostró el descenso asimétrico de las amígdalas del cerebelo, predominantemente del lado izquierdo, asociado con siringomielia que se extendía desde el nivel medular adyacente al espacio entre $\mathrm{C} 1$ y $\mathrm{C} 2$, hasta $\mathrm{T} 2$ (Figura 2). La resonancia magnética cerebral no demostró ninguna alteración.

Se realizó descompresión del foramen magnum, remoción del arco posterior de $\mathrm{C} 1$ y plastia de duramadre con injerto heterólogo. Tras un mes del procedimiento quirúrgico, el paciente refirió mejoría de la cefalea, sin embargo, las alteraciones tróficas, motoras y sensitivas de los músculos intrínsecos de la mano persisten. 


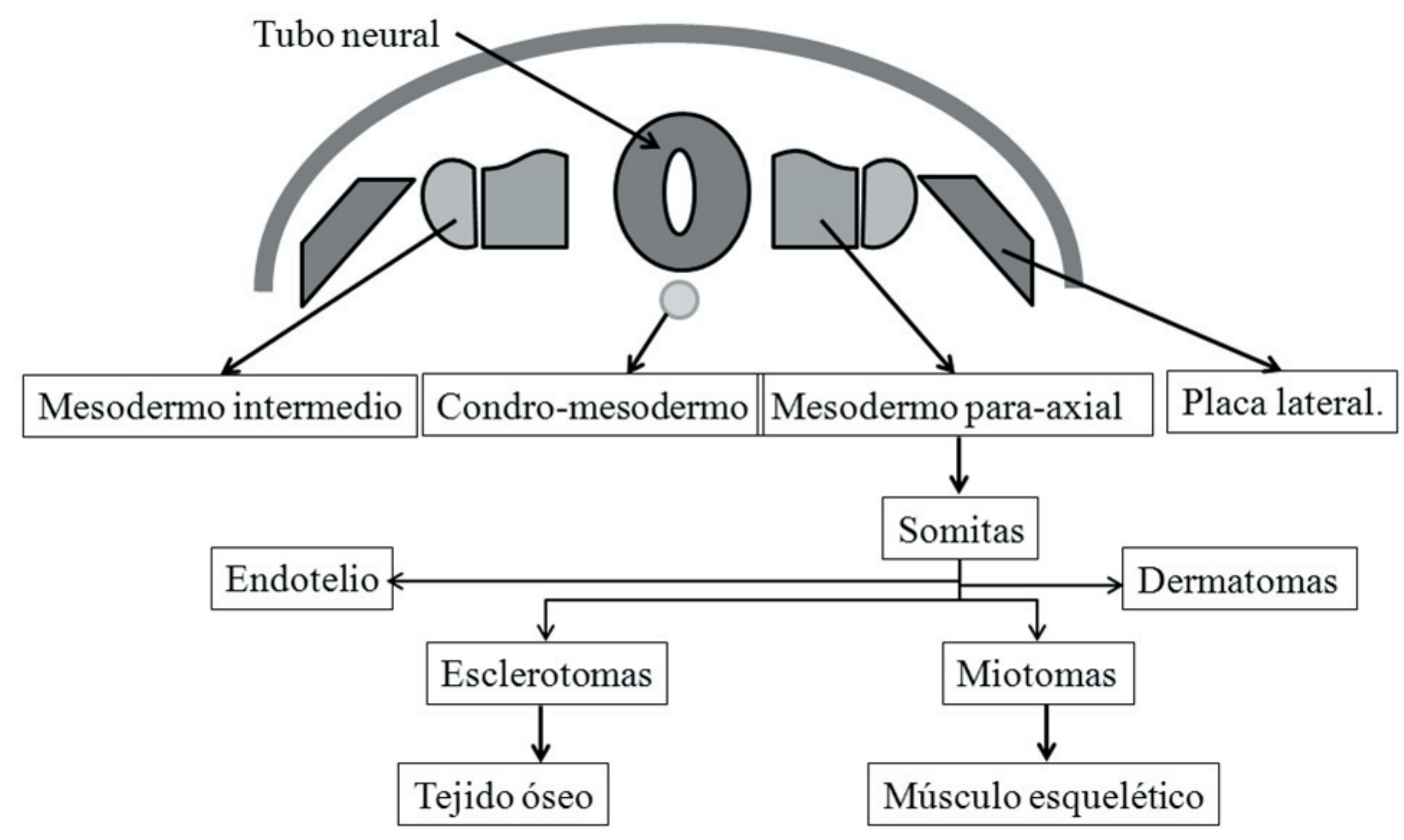

Figura 2. Desarrollo de las estructuras embriológicas involucradas en el desarrollo de anomalías mesodérmicas simultáneas.

\section{Discusión}

La MCHI y el SP podrían compartir algunos aspectos de su patogénesis. Tras la revisión de la literatura no fue encontrado ningún caso publicado previamente acerca de esta asociación, sin embargo, la coexistencia de la MCHI con otras anomalías de la porción para-axial del mesodermo apoyan la existencia de un trasfondo embriológico común ${ }^{17}$.

Ha sido demostrado que la proliferación acelerada del mesodermo embrionario motiva la aparición de tres subdivisiones: mesodermo lateral, intermedio y para-axial (inmediatamente adyacente al tubo neural ${ }^{4,21}$. Posteriormente el MPA y la notocorda crecen en sentido céfalocaudal (crecimiento longitudinal), dando origen a las somitas que conforman el primer esqueleto embrionario ${ }^{4,15,21}$. Inicialmente, 42 a 44 pares de somitas se distribuyen laterales a la notocorda, desde la proyección de la base del cráneo hasta la cola embrionaria. Las 12 más cefálicas formarán el occipucio, los huesos faciales, el oído interno y el resto de las estructuras mesodérmicas cervicales, incluyendo la musculatura voluntaria y la piel. Subsecuentemente, las somitas se diferencian en esclerotomas, sindotomas, células endoteliales, miotomas y dermatomas. Los esclerotomas, las primeras subdivisiones en aparecer, son los vestigios tanto de las vértebras como del hueso occipital ${ }^{15,21}$.

Los huesos occipitales humanos (basioccipital, occipital lateral y el planum nuchale de la escama) se desarrollan, como en el resto de los vertebrados, desde tres pares de vértebras occipitales rudimentarias, cuya naturaleza segmentada desaparece mediante su fusión ${ }^{15,20,21}$. La insuficiencia mesodérmica primaria ha sido considerada como la causa del crecimiento deficiente de la base del cráneo, especialmente del basioccipital, generando alteraciones del crecimiento del esqueleto axial, resultando en brevedad del segmento afectado y elevación progresiva de las placas neurales. Adicionalmente, puede disminuir la capacidad de ampliación de la notocorda, afectando al diámetro final del esqueleto axial del nivel afectado, que para el presente caso constituye la fosa posterior ${ }^{15}$.

Las malformaciones de las somitas occipitales en los pacientes con MCHI se manifiestan morfológicamente en diferentes alteraciones en la configuración de la base del cráneo $^{18,19}$. Varios estudios morfométricos describen diferentes grados de displasia de la basi-craneal, así como un menor volumen de la fosa posterior en comparación a los controles $^{19,22,24}$. Por su baja capacidad, la fosa posterior de los pacientes con MCHI sería insuficiente para contener al tallo cerebral y al cerebelo, deformándolo y promoviendo la herniación de las amígdalas cerebelosas a través del fora- 
men magnum ${ }^{17,19,23}$.

El SP ha sido catalogado clásicamente como la asociación de una deficiencia del músculo pectoral aunada a deformidades del miembro superior ipsilateral; sin embargo, actualmente se reconoce que las alteraciones aisladas del músculo pectoral también entran dentro del espectro del síndrome $e^{1,10,11}$. Con base en las manifestaciones clínicas, el SP puede ser clasificado en tres grupos: 1. SP leve o parcial, cuando solo está presente el defecto del músculo pectoral; 2. SP moderado o clásico, cuando exhibe deformidades ipsilaterales del tórax o la mano y sindactilia, y 3. SP grave, que incluye aplasia costal con herniación pulmonar, dextrocardia, hipoplasia muscular que se extiende hasta el latissimus dorsi y deltoides, ectrodactilia $y$ en algunos casos agenesia renal ${ }^{11}$.

La etiología del SP no ha sido bien esclarecida. Algunos autores postulan que una alteración del flujo sanguíneo embrionario puede ocasionar los defectos torácicos y del miembro superior ipsilateral (usualmente esclerodactilia); cuya extensión y gravedad estarían relacionadas con el sitio y el grado de alteración vascular. Se cree que la hipoplasia de la arteria torácica interna podría causar agenesia de la porción esterno-costal del pectoralis major, mientras que la hipoplasia de la arteria braquial podría conllevar deformidades de la mano ${ }^{10}$. En concordancia con esta explicación, existen varios reportes de la ocurrencia del SP con entidades relacionadas con disrupción vascular, entre ellas, el síndrome de Goldenhar, microsomía hemifacial atípica, anomalía de Sprengel, displasia aurículo-vertebral, displasia fronto-nasal, síndrome de Moebius, defectos del tubo neural y la anomalía de Klippel-Feil',5-8,12-14,17.

Bamforth y colaboradores señalan que todas las manifestaciones del SP son la consecuencia de una disrupción del mesodermo durante el periodo comprendido entre los días 16 y 28 posteriores a la fertilización ${ }^{3,10}$. Esto podría explicar tanto las alteraciones musculares como las arteriales, ya que ambas formaciones son derivadas del mesodermo.

Las teorías que explican la fisiopatología de la MCHI y el SP confluyen en alteraciones embriológicas muy tempranas del desarrollo del mesodermo para-axial, posiblemente en el período subsecuente la formación de las somitas, alrededor de la cuarta a la sexta semana de gestación.

\section{Conclusión}

La MCHI y el SP son dos patologías cuya coexistencia no había sido reportada previamente. Las alteraciones del desarrollo de las estructuras óseas que conforman la fosa posterior en los pacientes con MCHI han sido explicadas previamente por insuficiencia mesodérmica primaria, durante la formación del MPA, lo cual también ha sido descrito en el SP.
Este inusual caso, además de presentar por primera vez esta asociación, brinda mayor soporte a las teorías que vinculan al mesodermo para-axial como la causa de las alteraciones morfológicas de la fosa posterior de los pacientes con MCHI, en las que las alteraciones neurales serían el resultado de la adaptación a un continente anormalmente estrecho.

\section{Bibliografía}

1. Avci, G., Misirlioglu, A., Eker, G., Akoz, T.: Mild degree of Poland's Syndrome reconstruction with customized silicone prosthesis. Aesthetic Plast Surg 2003; 27: 112-115.

2. Baban, A., Torre, M., Bianca, S., et al.: Poland syndrome with bilateral features: case description with review of the literature. Am J Med Genet A 2009; 149A: 1597-1602.

3. Bamforth, J.S., Fabian, C., Machin, G., Honore, L.: Poland anomaly with a limb body wall disruption defect: case report and review. Am J Med Genet 1992; 43: 780-784.

4. Bess, S., Varma, V.: Embryology and Anatomy: Spine/ Spinal Cord. Embryology and Anatomy: Spine/Spinal Cord. En: Akbarnia, B.A., Yazici, M., Thompson, G.H. (ed). The Growing Spine. Management of Spinal Disorders in Young Children. Berlin; Springer-Verlag Berlin Heidelberg, 2011; pp. 3-12.

5. Coban, D., Gunes, T., Akin, M.A., Akcakus, M., Yikilmaz, A.: Poland anomaly and hydranencephaly: An unusual association. Am J Med Genet A 2010; 152A: 3182-3184.

6. Cobben, J.M., van Essen, A.J., McParland, P.C., Polman, H.A., ten Kate, L.P.: A boy with Poland anomaly and facio-auriculo-vertebral dysplasia. Clin Genet 1992; 41: 105107.

7. Derman, O., Gold, M.A.: Poland's syndrome and premature ovarian failure. J Pediatr Adolesc Gynecol 2004; 17 : 389-392.

8. Erol, M., Caksen, H., Tan, O., Atik, B., Unal, O., Odabas, D.: Report of a girl with Klippel-feil syndrome and Poland anomaly. Genet Couns 2004; 15: 469-472.

9. Fernández, A.A., Guerrero, A.I., Martínez, M.I., et al.: Malformations of the craniocervical junction (Chiari type I and syringomyelia: classification, diagnosis and treatment). BMC Musculoskelet Disord 2009; 10 Suppl 1: S1.

10. Fokin, A.A., Robicsek, F.: Poland's syndrome revisited. Ann Thorac Surg 2002; 74: 2218-2225.

11. Fokin, A.A.: Thoracic defects: cleft sternum and Poland syndrome. Thorac Surg Clin 2010; 20: 575-582.

12. Guion-Almeida, M.L., da Silva Lopes, V.L.: Frontonasal dysplasia, Poland anomaly and unilateral hypoplasia of lower limb: report on a male patient. Clin Dysmorphol 2003; 12: 233-236.

13. Hennekam, R.C., Hofstee, N.: Familial liability to intrauterine vascular impairments. Pediatrics 1990; 86: 326327. 
14. Kiratli, H., Erdener, U.: Poland-Moebius syndrome: a case report. Jpn J Ophthalmol 2000; 44: 679-682.

15. Marin-Padilla, M.: Notochordal-basichondrocranium relationships: abnormalities in experimental axial skeletal (dysraphic) disorders. J Embryol Exp Morphol 1979; 53: 1538.

16. Meadows, J., Kraut, M., Guarnieri, M., Haroun, R.I., Carson, B.S.: Asymptomatic Chiari Type I malformations identified on magnetic resonance imaging. J Neurosurg 2000; 92: 920-926.

17. Mesiwala, A.H., Shaffrey, C.I., Gruss, J.S., Ellenbogen, R.G.: Atypical hemifacial microsomia associated with Chiari I malformation and syrinx: further evidence indicating that chiari I malformation is a disorder of the paraaxial mesoderm. Case report and review of the literature. J Neurosurg 2001; 95: 1034-1039.

18. Milhorat, T.H., Chou, M.W., Trinidad, E.M., et al.: Chiari I malformation redefined: clinical and radiographic findings for 364 symptomatic patients. Neurosurgery 1999; 44: 1005-1017.

19. Nishikawa, M., Sakamoto, H., Hakuba, A., Nakanishi, N., Inoue, Y.: Pathogenesis of Chiari malformation: a morphometric study of the posterior cranial fossa. J Neurosurg 1997; 86: $40-47$.

20. O'Rahilly, R., Muller, F., Meyer, D.B.: The human vertebral column at the end of the embryonic period proper. 2 . The occipitocervical region. J Anat 1983; 136: 181-195.

21. Pang, D., Thompson, D.N.: Embryology and bony malformations of the craniovertebral junction. Childs Nerv Syst 2011; 27: 523-564.

22. Stovner, L.J., Bergan, U., Nilsen, G., Sjaastad, O.: Posterior cranial fossa dimensions in the Chiari I malformation: relation to pathogenesis and clinical presentation. Neuroradiology 1993; 35: 113-118.

23. Vannemreddy, P., Nourbakhsh, A., Willis, B., Guthikonda, B.: Congenital Chiari malformations. Neurol India 2010; 58: 6-14.

24. Vega, A., Quintana, F., Berciano, J.: Basichondrocranium anomalies in adult Chiari type I malformation: a morphometric study. J Neurol Sci 1990; 99: 137-145.

Alcalá-Cerra, G.; Sabogal-Barrios, R.; Niño-Hernández, L.M.; Gutiérrez-Paternina, J.J.; Moscote-Salazar, L.R.; Preciado-Mesa, E.: Malformación de Chiari tipo I en un paciente con síndrome de Poland. Neurocirugía 2011; 22: 583-587.

Correspondencia: Zaragocilla, Edificio Hospital Universitario del Caribe Calle 29 No $50-50$.

Correo electrónico: alcalagabriel@gmail.com 